\title{
Double-guidewire technique using a novel ERCP cannula for hepatic hilar obstruction
}

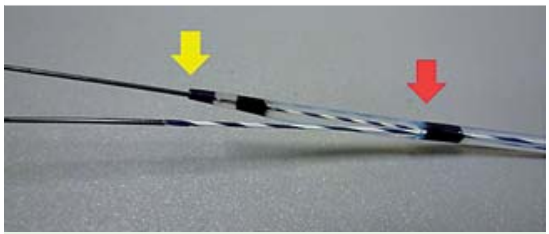

Fig. 1 The uneven double-lumen cannula with a 0.025 -inch guidewire lumen (yellow arrow) and a 0.035-inch guidewire lumen (red arrow).

Endoscopic biliary drainage (EBD) for hepatic hilar obstruction is sometimes challenging. Moreover, when unilateral biliary drainage has already been performed, if another intrahepatic bile duct also needs to be drained, EBD may be made more difficult because of tumor ingrowth and the narrowed angle from which the bile duct must be accessed. In this case, percutaneous biliary drainage (PTBD) or endoscopic ultrasound-guided biliary drainage (EUS-BD), especially EUS-guided hepaticogastrostomy (EUS-HGS), may be indicated. However, PTBD is known to be associated with several adverse events such as cholangitis, biliary leakage, and pneumothorax [1], and EUS-HGS is associated with adverse events such as bile peritonitis, cholangitis, and potentially fatal stent dysfunction [2]. It appears that EBD may be more feasible compared with these other salvage techniques.

Recently, a novel endoscopic retrograde cholangiopancreatography (ERCP) cannula with an uneven double lumen has been made available (Piolax Medical Devices, Kanagawa, Japan) ( Fig.1). Herein, we present a "double-guidewire technique" using this novel ERCP cannula for hepatic hilar obstruction.

A 45-year-old man was referred to our hospital with obstructive jaundice due to left intrahepatic bile duct obstruction. He had undergone EBD 6 months previously for relief of obstructive jaundice caused by pancreatic cancer with an uncovered metal stent being placed between the right hepatic duct and the common bile duct and a fully covered metal stent being placed between the upper and lower bile ducts. We decided that he would need reintervention to treat the obstructed left intrahepatic bile duct.
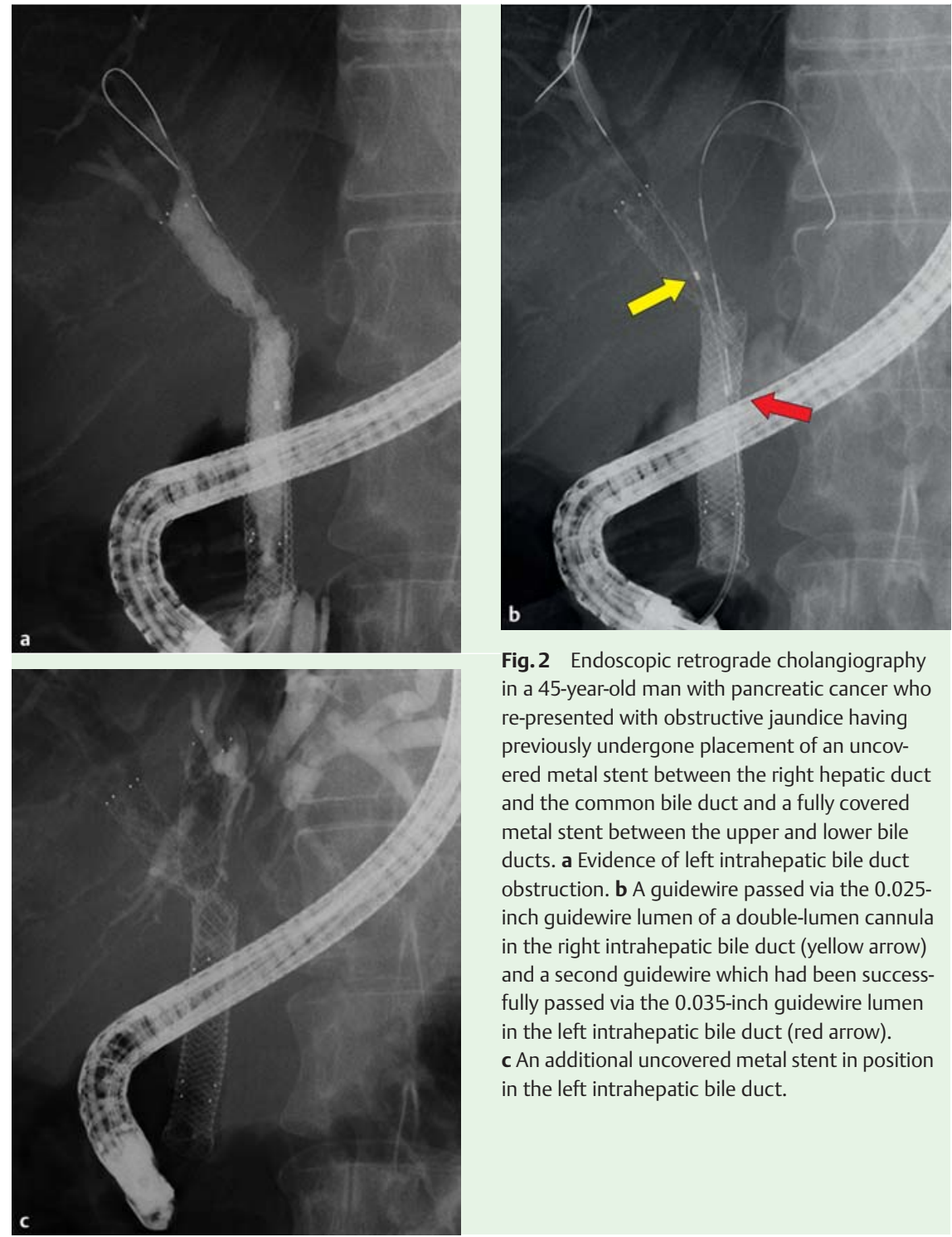

Fig. 2 Endoscopic retrograde cholangiography in a 45-year-old man with pancreatic cancer who re-presented with obstructive jaundice having previously undergone placement of an uncovered metal stent between the right hepatic duct and the common bile duct and a fully covered metal stent between the upper and lower bile ducts. a Evidence of left intrahepatic bile duct obstruction. b A guidewire passed via the 0.025 inch guidewire lumen of a double-lumen cannula in the right intrahepatic bile duct (yellow arrow) and a second guidewire which had been successfully passed via the 0.035 -inch guidewire lumen in the left intrahepatic bile duct (red arrow). c An additional uncovered metal stent in position in the left intrahepatic bile duct.

Firstly, we inserted a standard ERCP cannula (MTW Endoscopy, Düsseldorf, Germany) into the previously placed metal stent and injected contrast medium, which confirmed the left intrahepatic bile duct obstruction ( $\bullet$ Fig.2a). An attempt was then made to pass a 0.025 -inch guidewire (VisiGlide; Olympus Medical Systems, Tokyo, Japan) through the left intrahepatic bile duct obstruction, but this was unsuccessful. We therefore changed the cannula to the new uneven double-lumen cannula, advancing this first into the right intrahepatic bile duct with a guidewire through the 0.025-inch guidewire lumen.
The cannula was then slowly withdrawn while we probed for the left intrahepatic bile duct using a guidewire (Cheer Leader+M, angle type; Tri-Med Co. Ltd., Osaka, Japan) via the 0.035-inch lumen and we were able to successfully advance the guidewire through the left intrahepatic bile duct obstruction ( $\bullet$ Fig. 2 b; - Video 1).

Finally, once the guidewire had been placed in the left intrahepatic duct, we inserted an uncovered metal stent between the left intrahepatic duct and the common bile duct ( $\bullet$ Fig. 2c). No complications were observed, and the jaundice resolved 


\section{Video 1}

Fluoroscopy during endoscopic retrograde cholangiography in a 45-year-old man with pancreatic cancer and obstructive jaundice showing that we were unable to advance a guidewire into the obstructed left intrahepatic bile duct using a standard cannula. However we were able to do so with a double-lumen cannula that had been advanced into the right intrahepatic duct and was then slowly withdrawn while we probed for the left intrahepatic bile duct using a guidewire inserted through the 0.035 -inch guidewire lumen. Once a guidewire had been successfully advanced into the left intrahepatic bile duct, an uncovered metal stent was then successfully placed over it.

postoperatively. The double-guidewire technique using an uneven double-lumen cannula may be of clinical value when trying to locate and pass a guidewire through an obstruction at the hepatic hilum, as presented in this case.
Endoscopy_UCTN_Code_TTT_1AR_2AZ

Competing interests: None

\section{Takeshi Ogura, Daisuke Masuda, Akira Imoto, Rieko Kamiyama, Eiji Umegaki, Kazuhide Higuchi}

Second Department of Internal Medicine, Osaka Medical College, Osaka, Japan

\section{References}

1 Oh HC, Lee SK, Lee TY et al. Analysis of percutaneous transhepatic cholangioscopy-related complications and the risk factors for those complications. Endoscopy 2007; 39: $731-736$

2 Martin FP, Rossini LG, Ferrari AP. Migration of a covered metallic stent following endoscopic ultrasound-guided hepaticogastrostomy: fatal complication. Endoscopy 2010; 42: E126-E127

\section{Bibliography}

Dol http://dx.doi.org/ 10.1055/s-0033-1359166

Endoscopy 2014; 46: E56-E57

(c) Georg Thieme Verlag KG

Stuttgart · New York

ISSN 0013-726X

\section{Corresponding author}

Takeshi Ogura, MD, PhD

Second Department of Internal Medicine Osaka Medical College

2-7 Daigaku-machi

Takatsuki-shi

Osaka 569-8686

Japan

Fax: +81-72-6846532

oguratakeshi0411@yahoo.co.jp 